\title{
HEAT TRANSFER BEHAVIOUR AND FLOW FIELD CHARACTERISATION OF IMPINGING SYNTHETIC JETS FOR A WIDE RANGE OF PARAMETERS
}

\author{
Rayhaan Farrelly \\ Trinity College Dublin \\ Dublin, Ireland, farrelra@tcd.ie \\ Tim Persoons \\ Trinity College Dublin \\ Dublin, Ireland
}

\author{
Alan McGuinn \\ Trinity College Dublin \\ Dublin, Ireland \\ Darina Murray \\ Trinity College Dublin \\ Dublin, Ireland
}

\begin{abstract}
Impinging synthetic jets are considered as a potential solution for convective cooling, in applications that match their main characteristics (high local heat transfer rates, zero net mass flux, scalability, active control). Nevertheless the understanding of heat transfer to synthetic jets falls short of that available for steady jets. To address this, this paper uses detailed flow field measurements to help identify the main heat transfer mechanisms in impinging synthetic jets.

Local heat transfer measurements have been performed for an impinging round synthetic jet at a range of Reynolds numbers between 1000 and 3000, nozzle to plate spacings between $4 D$ and $16 D$ and stroke lengths $\left(L_{0}\right)$ between $2 D$ and $32 D$. The heat transfer results show evidence of distinct regimes in terms of $L_{0} / D$ and $L_{0} / H$ ratios. Based on appropriate scaling, four heat transfer regimes are identified which justifies a detailed study of the flow field characteristics.

High speed particle image velocimetry (PIV) has been employed to measure the time-resolved velocity flow fields of the synthetic jet to identify the flow structures at selected $L_{0} / H$ values corresponding to the identified heat transfer regimes. The flow measurements support the same regimes as identified from the heat transfer measurements and provide physical insight for the heat transfer behaviour.
\end{abstract}

\section{INTRODUCTION}

A synthetic jet is a fluid motion consisting of a train of propagating vortices, generated by a sufficiently strong oscillatory flow at a sudden expansion. When impinging on a heated surface, the vortices affect the heat transfer by enhancing the transport and mixing of the heated fluid. The main variables for synthetic jet impingement heat transfer are the dimensionless stroke length $L_{0} / D$, the jet Reynolds number $R e=\rho U_{0} D / \mu$ and the dimensionless jet exit to impingement surface $(H / D)$. Impinging synthetic jets have recently shown promise in various practical applications, in particular in cooling applications with confined geometries. It has been shown by Kercher et al. (2003) that synthetic jets can deliver similar cooling effects without the need for an external air supply system. In a review article by Glezer and Amitay (2002) it was noted that impinging synthetic jets are proving to be an extremely promising technology for use in electronics cooling. Electronics densification is the current industry objective, and it has been established that there is a need for more efficient cooling methods in these closely packed systems, with this need continuing to grow in the foreseeable future, Black et al. (1998).

It is well established that effective rates of cooling can be achieved using conventional steady flow impinging jets; Gardon and Akfirat (1965), Hollworth and Wilson (1984) and Lyttle and Webb (1994). The synthetic impinging jet can be easily integrated into complex geometries, requires low operating power and is relatively simple to design and fabricate, and, unlike a steady impinging jet, it does not require external ducting due to its zero net mass flux nature.

Previous investigations into the heat transfer measurements of a synthetic jet at $\mathrm{H} / \mathrm{D}<4$ have been carried out by McGuinn et al. (2009). Shuster and Smith (2007) investigated the formation and convection of a round impinging synthetic jet; 
three flow regimes were mapped for a synthetic jet with a range of parameters and they also presented dye-flow visualisations showing little or no jet formed below a threshold stroke length of $\mathrm{L}_{0} / \mathrm{D}=2$.

Many authors including Gillespie et al. (2006), Valiorgue et al. (2008) and Pavlova and Amitay (2006) have presented PIV flow visualisation data for an impinging synthetic jet flow field with corresponding heat transfer data. However Valiorgue et al. (2008) presented results at $\mathrm{H} / \mathrm{D}=2$ and $\mathrm{L}_{0} / \mathrm{D}$ values up to 10. Pavlova and Amitay (2006) did not present heat transfer profiles.

Due to the complex flow characteristics of an impinging synthetic jet, the relationships between flow parameters such as dimensionless stroke length, Reynolds number and jet to plate spacing and the resulting heat transfer have yet to be fully investigated and understood. As such this paper investigates the flow field of an impinging axisymmetric synthetic jet using high-speed PIV to help explain its convective heat transfer characteristics, and in particular, to identify different regimes in this complex flow configuration.

\section{NOMENCLATURE}

D Jet diameter, $\mathrm{m}$

h Convective heat transfer co-efficient, $\mathrm{W} /\left(\mathrm{m}^{2} \mathrm{~K}\right)$

$\mathrm{H} \quad$ Height of nozzle above plate, $\mathrm{m}$

$\mathrm{k}$ Thermal conductivity, $\mathrm{W} /(\mathrm{mK})$

$\mathrm{k}_{\mathrm{s}} \quad$ Thermal conductivity of sensor barrier, $\mathrm{W} /(\mathrm{mK})$

$\mathrm{L}_{\mathrm{o}} / \mathrm{D} \quad$ Jet dimensionless stroke length

$\mathrm{Nu} \quad$ Nusselt number, based on $\mathrm{D}$ and jet cavity temperature

Pr Prandtl number

$\ddot{q} \quad$ Heat flux, $\mathrm{W} / \mathrm{m}^{2}$

$\mathrm{r} \quad$ Radial distance along plate from geometric centre, $\mathrm{m}$

Re Reynolds number, based on D

$\Delta \mathrm{T} \quad$ Temperature difference across sensing element, ${ }^{\circ} \mathrm{C}$

$\mathrm{T}_{\mathrm{s}} \quad$ Temperature of heated surface, ${ }^{\circ} \mathrm{C}$

$\mathrm{T}_{\text {cavity }} \quad$ Temperature of cavity, ${ }^{\circ} \mathrm{C}$

$\mathrm{t}_{\mathrm{s}} \quad$ Light sheet thickness, $\mathrm{m}$

$\mathrm{U}_{\mathrm{o}} \quad$ Average jet velocity, $\mathrm{m} / \mathrm{s}$

$\mathrm{U}_{\mathrm{RMS}} \quad$ Root mean squared jet velocity, $\mathrm{m} / \mathrm{s}$

$\delta \quad$ Sensor thickness, $\mathrm{m}$

$v \quad$ Kinematic viscosity, $\mathrm{m}^{2} / \mathrm{s}$

\section{EXPERIMENTAL SETUP}

Three main elements make up the experimental rig, (i) the synthetic jet assembly, (ii) the isothermally heated impingement surface with embedded instrumentation and (iii) the particle image velocimetry system. The impingement surface and the jet are mounted on independent carriages that travel on orthogonal tracks; the carriage for the impingement surface is moved using a computer controlled traverse, whereas the carriage for the jets is moved using a manually operated lead screw actuator. The instruments associated with the heated impingement surface are two single point heat flux sensors and two thermocouples. The rig design is presented in figure 1 .

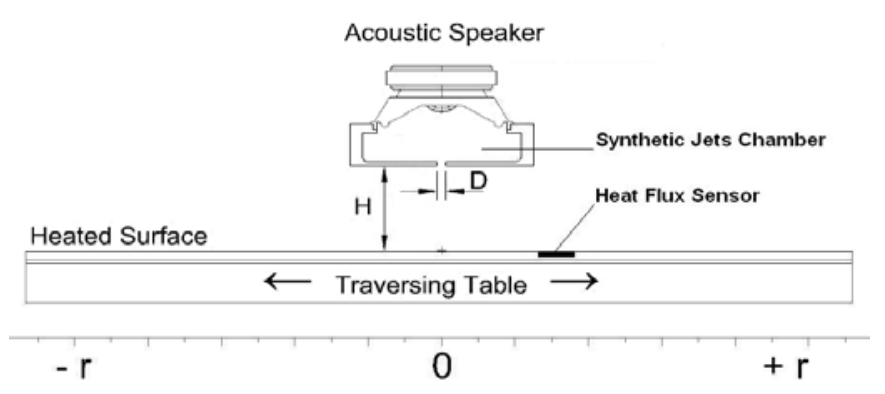

Figure 1: Schematic of the synthetic jet test apparatus

The operation of the synthetic jet relies primarily on an acoustic speaker mounted on an enclosed cavity with an orifice plate on the opposing side to provide the entrainment path for the working fluid. The speaker is a Visaton ${ }^{\circledR}$ FR8 audio speaker. A signal input is required to control the driving frequency of the speaker; this is provided by a TTi TG315 Signal Generator, and the signal is amplified using a Kemo ${ }^{\circledR} 40$ Watt power amplifier. The speaker was supplied with a sinusoidal wave of specific amplitude and frequency so as to obtain the desired stroke length and Reynolds number. The use of a loudspeaker allows for a more continuous range of driving frequencies than has been achieved in the past. The voice coil is cooled using air jets in an enclosed chamber, to minimise parasitic heating of the air within the synthetic jet cavity.

The cavity body and orifice plate are constructed from a thermoplastic and were fabricated using a method of rapid prototyping called fused deposition modelling. The cavity has an approximate volume of $100 \mathrm{~cm}^{3}$ with an orifice diameter of $5 \mathrm{~mm}$. The synthetic jet is clamped onto a carriage, which allows for height adjustment from 1 to 16 diameters above the impingement surface. The jet is fixed at a normal angle of impingement $\left(90^{\circ}\right)$.

An impinging synthetic jet flow is characterised by three parameters: The surface to orifice spacing $H / D$, the dimensionless stroke length $\mathrm{L}_{0} / \mathrm{D}$ and the Reynolds number $R e=U_{0} D / v$, where $L_{0}=\int_{0}^{T / 2} U(t) \mathrm{d} t, \mathrm{~T}$ is the oscillation period, $\mathrm{U}_{0}=\mathrm{L}_{0} /(\mathrm{T} / 2)$ and $\mathrm{U}(\mathrm{t})$ is the area-averaged orifice velocity. $\mathrm{L}_{0} / \mathrm{D}$ is inversely proportional to a Strouhal number, since $L_{0} / D=\frac{1}{2}\left(f D / U_{0}\right)^{-1}$. The synthetic jet operating point is set using a semi-empirical relationship between the cavity pressure and jet velocity with an orifice damping coefficient $\mathrm{K}=1.459$, Persoons and O'Donovan (2007). 
Table 1: Synthetic Jet Test Parameters

\begin{tabular}{|c|c|c|c|}
\hline $\begin{array}{l}\text { Reynolds } \\
\text { Number }\end{array}$ & $\mathbf{1 0 0 0}$ & $\mathbf{2 0 0 0}$ & $\mathbf{3 0 0 0}$ \\
\hline $\mathrm{L}_{0} / \mathrm{D}$ & Frequency (Hz) & Frequency $\mathbf{( H z )}$ & Frequency (Hz) \\
\hline 30 & 10 & 21 & 31 \\
\hline 20 & 15 & 30 & 46 \\
\hline 16 & 19 & 38 & 58 \\
\hline 12 & 26 & 51 & 77 \\
\hline 10 & 31 & 61 & 92 \\
\hline 8 & 38 & 76 & 116 \\
\hline 6 & 51 & 101 & 154 \\
\hline 4 & 77 & 150 & 232 \\
\hline 3 & 100 & 200 & 308 \\
\hline 2 & 150 & 312 & 460 \\
\hline
\end{tabular}

The jet impinges onto a surface that consists of a $5 \mathrm{~mm}$ thick flat copper plate measuring $425 \mathrm{~mm} \times 550 \mathrm{~mm}$. To the underside of the plate a silicon rubber heater mat is glued with a thin layer of adhesive. The mat is approximately $1.1 \mathrm{~mm}$ thick. The underside of the plate and mat assembly is insulated from the surroundings. The entire assembly is such that it approximates a uniform wall temperature boundary condition. The system is typically operated at a surface temperature of $40^{\circ} \mathrm{C}$.

The sensor utilised in this study is an RdF Micro-Foil ${ }^{\circledR}$ heat flux sensor. The heat flux sensor is mounted flush with the surface of the plate; to achieve this it was necessary to embed the sensor in the plate by machining a groove in the surface and gluing it down using a thermally conductive epoxy. The sensor is positioned centrally on the plate, and together with the jet and plate carriage arrangement, allow for heat transfer measurements beyond 20 diameters from the geometric centre of the jet. In this study, testing has only been concerned within the region of 1 to 6 diameters from the geometric centre of the jet. The Micro-Foil ${ }^{\mathbb{B}}$ heat flux sensor measures the temperature differential across a known thermal barrier using a differential thermopile. The heat flux through the sensor is based on the following equation:

$$
\ddot{q}=k_{s} \frac{\Delta T}{\delta}
$$

Where $\Delta \mathrm{T}$ is the temperature difference across the thickness $(\delta)$ of the barrier and $\mathrm{k}_{\mathrm{s}}$ is the thermal conductivity of the barrier (kapton). A single pole T-type thermocouple is also embedded in this sensor to measure the local temperature.

The Micro-Foil ${ }^{\circledR}$ heat flux sensor was calibrated using a contoured nozzle with a diameter of $13 \mathrm{~mm}$. For a round unconfined steady impinging jet, Liu and Sullivan (1996) have shown that at low H/D $(<2)$ the convective heat transfer coefficient at the stagnation point is independent of nozzle height and is predicted well by the following correlation for Re between 10000 and 30000

$$
N u_{\text {stag }}=0.585 \operatorname{Re}^{0.5} \operatorname{Pr}^{0.4}
$$

The voltage produced by the Micro-Foil ${ }^{\circledR}$ heat flux sensor was recorded when the sensor was placed at the stagnation point under the impinging jet. The height of the nozzle above the heated impingement surface was $0.75 \mathrm{D}$. The Reynolds number was varied from 10000 to 30000 . The calculated heat flux was plotted against the voltage produced under the test conditions, which produced an $\mathrm{R}^{2}$ value of 0.9975 . The heat transfer co-efficient is calculated using the following equation:

$$
h=\frac{\ddot{q}}{\left(T_{s}-T_{\text {cavity }}\right)}
$$

and the corresponding Nusselt number (equation 4) is determined with the jet temperature as a reference.

$$
N u=h D / k
$$

The heat transfer rig used in this paper is similar to that used by O’Donovan and Murray (2007).

Flow measurements have been performed using Particle Image Velocimetry (PIV). The PIV system comprises of a Quantronix Darwin-Duo Nd:YLF twin cavity laser (up to 15 $\mathrm{mJ}$ at $1000 \mathrm{~Hz}, 527 \mathrm{~nm}$ ) and a LaVision HighSpeedStar6 CMOS camera $(1024 \times 1024$ pixels, 12 bit $)$ with a Sigma 50 $105 \mathrm{~mm}$ lens. A glycol-water aerosol is used for seeding, with particle diameters between 0.2 and $0.3 \mu \mathrm{m}$.

Several steps were taken to minimise reflection when performing PIV measurements on the synthetic jet. To avoid reflection on the orifice plate and the impingement surface, (a) the light sheet thickness is reduced to approximately, $t_{\mathrm{s}}=1 \mathrm{~mm}$ across the viewing window, (b) the orifice plate was painted fluorescent orange, (c) the impingement surface was painted matt black, and (d) a narrow band pass filter that allows only green light into the camera lens was utilised. The camera was tilted slightly to approximately $+2^{\circ}$ to further reduce reflections from the impingement surface. Perspective distortion was calibrated using LaVision's Davis software.

The velocity fields recorded have been processed with LaVision's Davis 7.2.2 software using multi-grid crosscorrelation with an interrogation window size decreasing from $64 \times 64$ to $32 \times 32$ pixels at $50 \%$ overlap, with further processing of the PIV flow visualisation plots in MATLAB. 


\section{RESULTS AND DISCUSSION}

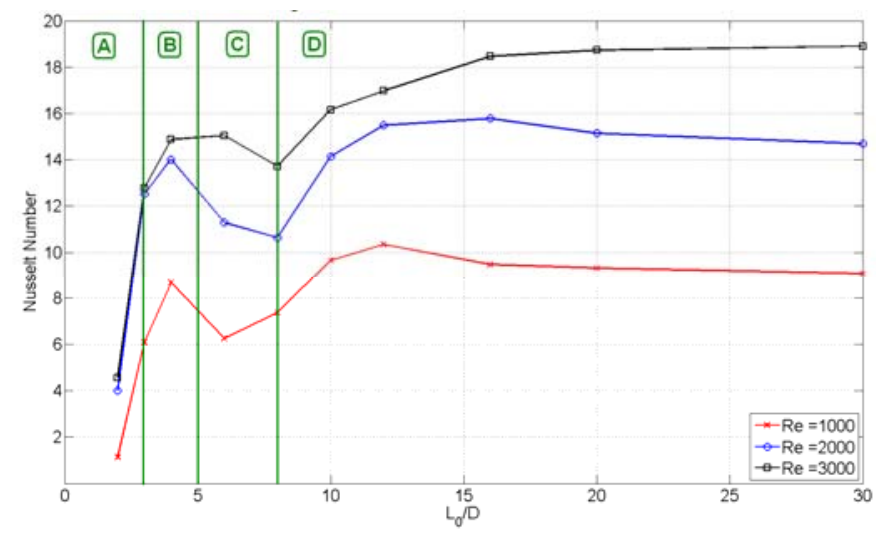

Figure 2: Stagnation point Nusselt number for impinging synthetic jet at $H / D=$ 4, with four heat transfer regimes (see Table 2)

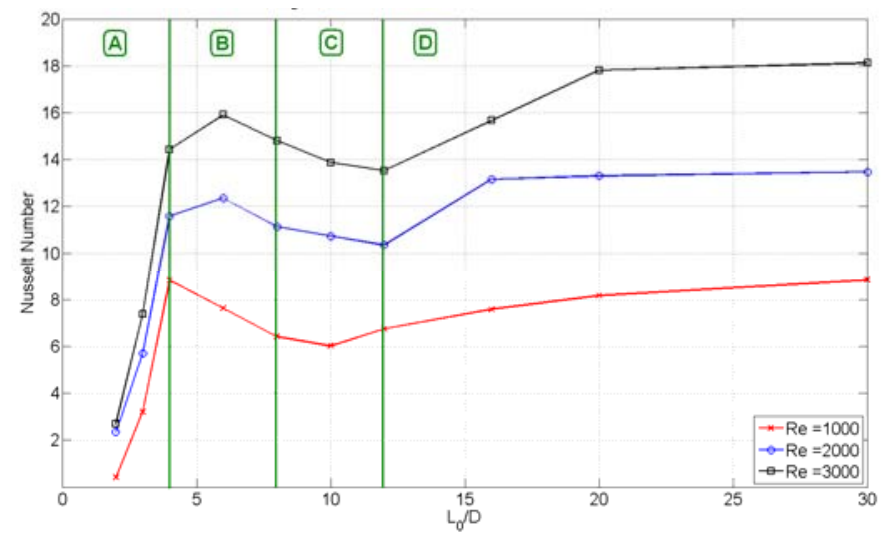

Figure 3: Stagnation point Nusselt number for impinging synthetic jet at $H / D=$ 8 , with four heat transfer regimes (see Table 2)

Table 2: Identified heat transfer regimes

\begin{tabular}{|c|c|c|c|}
\hline Regime & $\mathrm{H}$ & $\mathrm{L}_{0}$ & Description \\
\hline \multirow{2}{*}{ A } & 4D (Fig 2) & \multirow{2}{*}{$\mathrm{L}_{0} \leq 4 \mathrm{D}$} & \multirow{2}{*}{ Increasing heat transfer } \\
\hline & 8D (Fig 3) & & \\
\hline \multirow{2}{*}{ B } & 4D (Fig 2) & $\mathrm{L}_{0} \approx 4 \mathrm{D}$ & \multirow{2}{*}{ Peak heat transfer. } \\
\hline & 8D (Fig 3) & $\mathrm{L}_{0} \approx 6 \mathrm{D}$ & \\
\hline \multirow{2}{*}{$\mathrm{C}$} & 4D (Fig 2) & $\mathrm{L}_{0} \approx 8 \mathrm{D}$ & \multirow{2}{*}{ Local minimum in heat transfer. } \\
\hline & 8D (Fig 3) & $\mathrm{L}_{0} \approx 12 \mathrm{D}$ & \\
\hline \multirow{2}{*}{$\mathrm{D}$} & 4D (Fig 2) & $\mathrm{L}_{0}>8 \mathrm{D}$ & \multirow{2}{*}{ Increase in heat transfer. } \\
\hline & 8D (Fig 3) & $\mathrm{L}_{0}>12 \mathrm{D}$ & \\
\hline
\end{tabular}

Figures 2 and 3 represent stagnation point heat transfer plots for $H / D=4$ and $H / D=8$ respectively. They identify four regimes of interest from a heat transfer perspective. The lowest $\mathrm{L}_{0} / \mathrm{D}$ where significant heat transfer was recorded is similar to results presented by Valiorgue et al. (2008) for $H / D=2$, however in that study two distinct flow and heat transfer regimes were identified for the impinging synthetic jet, along with a critical stroke length of $\mathrm{L}_{0} / \mathrm{D}=5$.

Regime $\mathrm{A}$ in figures 2 and 3 indicate that jet formation becomes erratic below $\mathrm{L}_{0} / \mathrm{D} \approx 3$. The vortex ring generally does not escape the nozzle and becomes re-entrained during the suction phase of the cycle; as such no significant flow impinges upon the heated surface resulting in low heat transfer; these results correspond with findings by Valiorgue et al. (2008).

As stroke length $\left(\mathrm{L}_{0} / \mathrm{D}\right)$ increases (regime $\mathrm{B}$ ) the vortices generated travel a greater distance before the suction phase of the cycle occurs, allowing them to escape the reingestion zone. Increased vortex interaction upon impingement leads to the relatively high level of heat transfer observed in this regime.

The reduction in heat transfer seen in regime $\mathrm{C}$ has been attributed to a decrease in turbulence due to the reduced frequency of vortices prior to impingement resulting in less turbulent mixing at the heated surface. Regime D illustrates a region of greater heat transfer; this is considered to be due to a higher peak velocity experienced within the core flow caused by increased slug coherence.

From these stagnation point heat transfer plots alone it is difficult to infer the convective heat transfer mechanisms of the impinging synthetic jets. Therefore, profiles of local heat transfer coefficient are also presented. Furthermore, particle image velocimetry (PIV) was employed to aid in the analysis of these flow regimes to facilitate the understanding of what is occurring in the heat transfer plots presented.

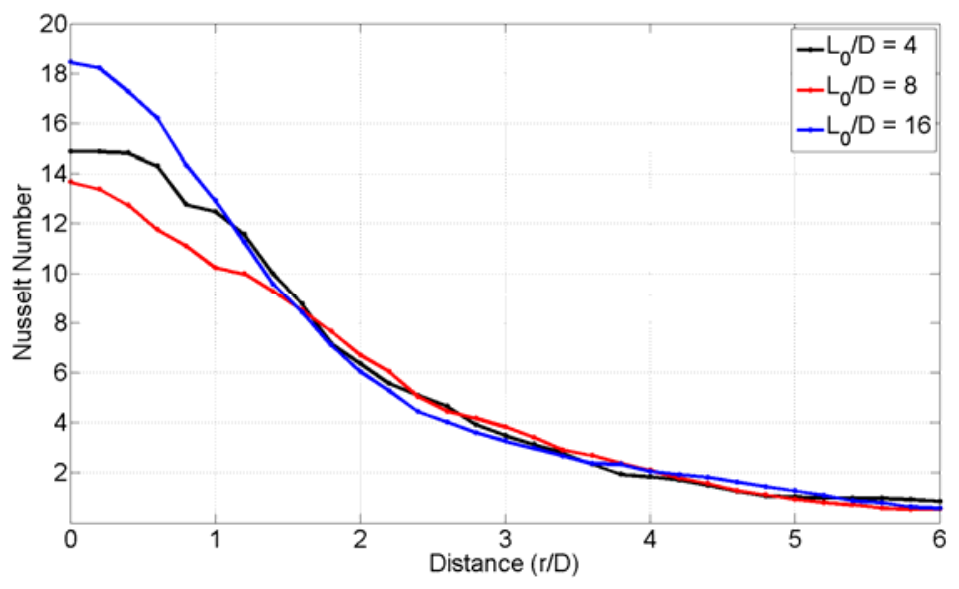

Figure 4: Radial profile of local Nusselt number for impinging synthetic jet at $\mathrm{H} / \mathrm{D}=4$ and $\mathrm{Re}=3000$ 


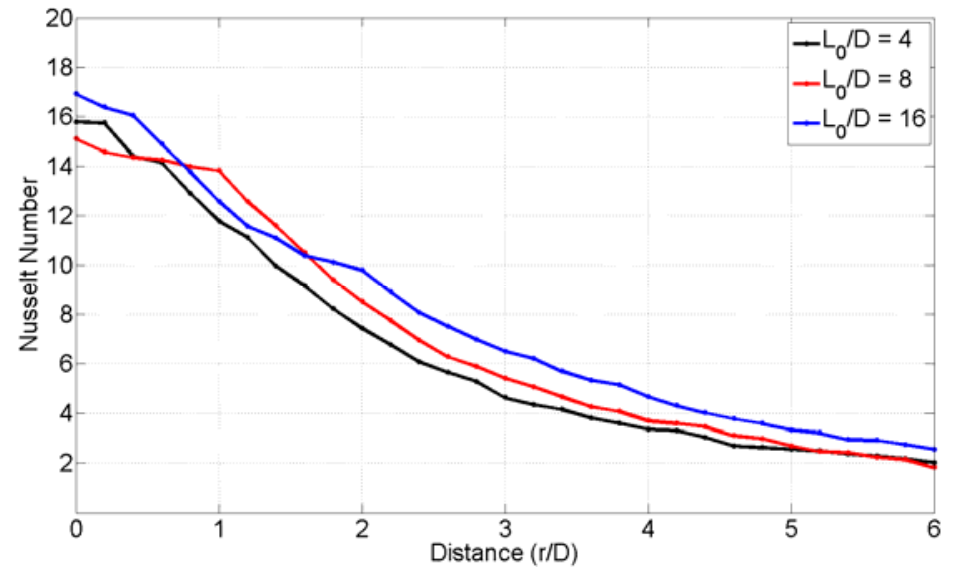

Figure 5: Radial profile of local Nusselt number for impinging synthetic jet at $\mathrm{H} / \mathrm{D}=8$ and $\mathrm{Re}=3000$
Figures 4 and 5 illustrate the radial heat transfer profiles of a synthetic jet for three different $\mathrm{L}_{0} / \mathrm{D}$ values of $4,8,16$, and for a jet Reynolds number of 3000 . Values presented show an overall maximum at the stagnation point, with local heat transfer decreasing with increasing radial distance. This trend was observed in all cases.

Figure 4 corresponds with the stagnation point heat transfer plot presented in figure 2 for a jet Reynolds number of 3000. Each radial profile coincides with a specific regime, i.e. the radial profile $\mathrm{L}_{0} / \mathrm{D}=4$ lies within regime $\mathrm{B}$. It is evident from these profiles that increasing the $\mathrm{L}_{0} / \mathrm{D}$ can effect greater heat transfer. Likewise the vortex interaction caused by smaller stroke lengths results in a lower yet broader radial Nusselt number profile.

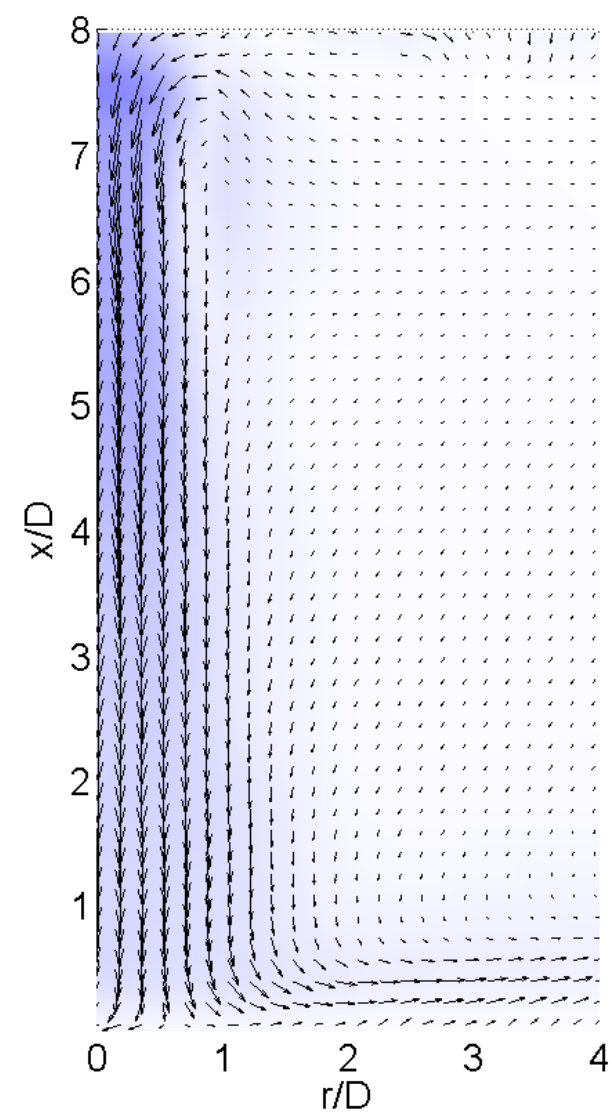

Figure 6: Time averaged velocity and turbulence intensity distribution of impinging synthetic jet $\left(\mathrm{H} / \mathrm{D}=8, \mathrm{~L}_{0} / \mathrm{D}=4, \mathrm{Re}=3000\right.$, Regime B)

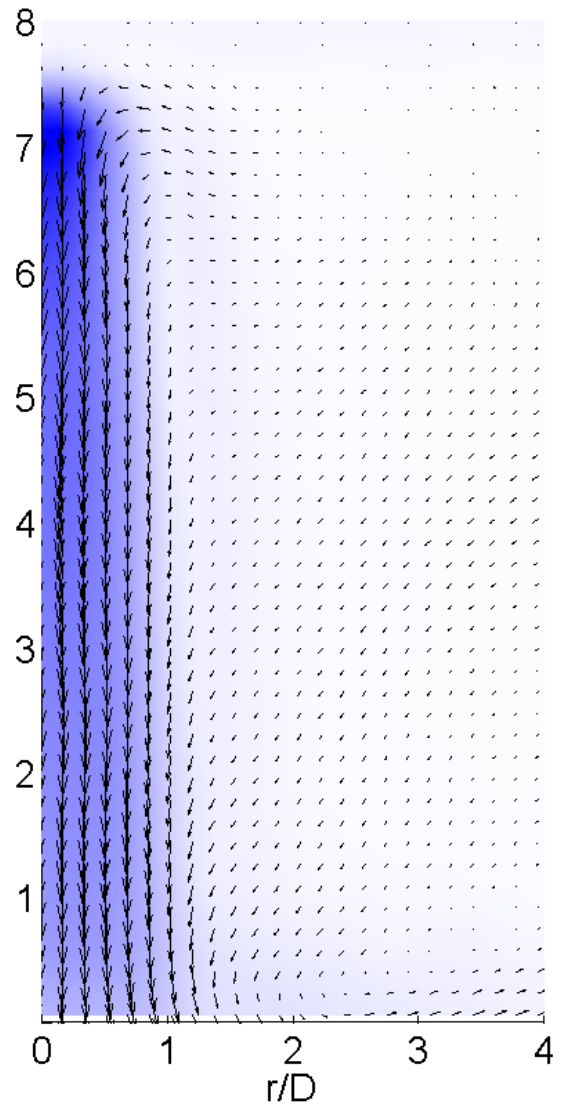

Figure 7: Time averaged velocity and turbulence intensity distribution of impinging synthetic jet $\left(\mathrm{H} / \mathrm{D}=8, \mathrm{~L}_{0} / \mathrm{D}=8, \mathrm{Re}=3000\right.$, Regime $\left.\mathrm{C}\right)$

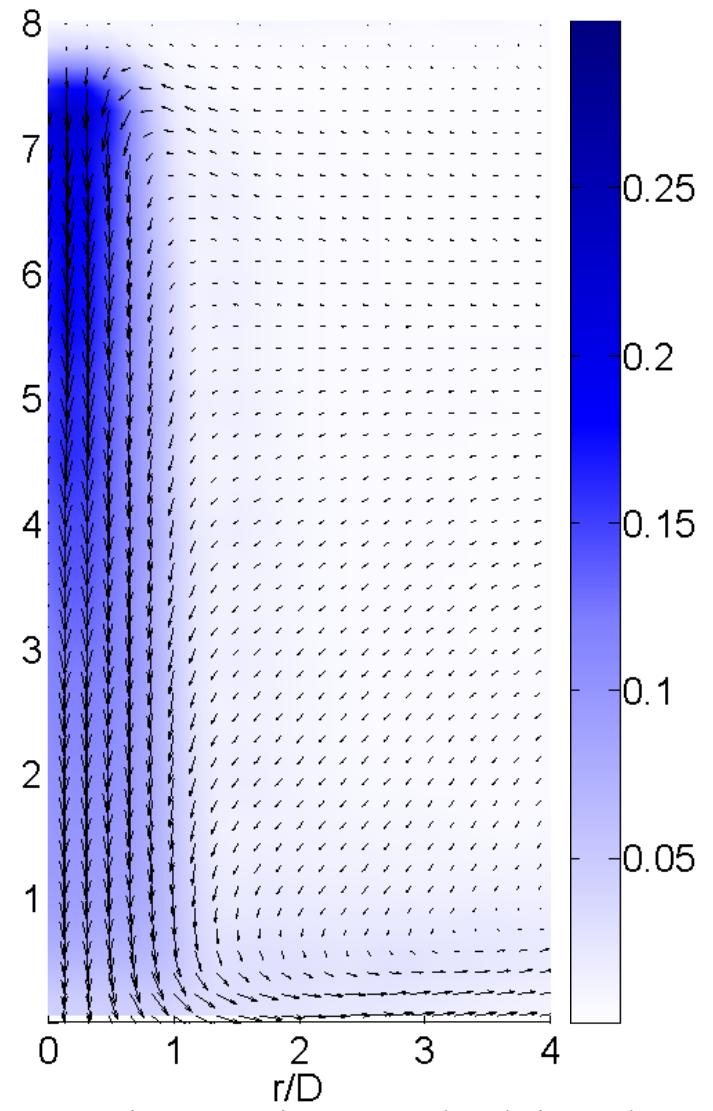

Figure 8: Time averaged velocity and turbulence intensity distribution of impinging synthetic jet $\left(\mathrm{H} / \mathrm{D}=8, \mathrm{~L}_{0} / \mathrm{D}=16, \mathrm{Re}=3000\right.$, Regime D)
For the sake of clarity in the phase locked Particle Image Velocimetry plots presented only 1 of every 2 vectors in the vertical direction has been plotted. The overall result makes the plot easier to interpret.
Figures 6 through 8 represent the time averaged flow field and turbulence intensity of the synthetic jet at $H / D=8, R e=3000$ and three different stroke lengths $\left(\mathrm{L}_{0} / \mathrm{D}\right) 4,8$ and 16 . The colour bar on the right hand side of figure 8 corresponds to turbulence intensity, $\mathrm{U}_{\mathrm{RMS}}{ }^{2} / \mathrm{U}_{0}^{2}$. 
In figure 6 for $\mathrm{L}_{0} / \mathrm{D}=4$, there is a lower turbulence intensity experienced within the jet core. At this low stroke length there are a greater number of vortices generated because of the higher driving frequency. As can be seen in figure 11, the vortices convect away from the nozzle, increased interaction results in vortex breakdown and an overall reduction in turbulence intensity. Increasing the stroke length $\left(\mathrm{L}_{0} / \mathrm{D}\right)$ to 8 in figure 7, it can be seen that that the turbulence intensity increases. This increase is particularly evident within the core flow of the jet. The reduced mixing is a direct result of the larger stroke length and the reduced driving frequency producing less vortical interactions upon impingement. This can be seen in figure 12. Figure 8 displays the greatest turbulence intensity for this $\mathrm{H} / \mathrm{D}$ and jet Reynolds number. A relatively large stroke length $\left(\mathrm{L}_{0} / \mathrm{D}=16\right)$ and low actuating frequency result in a slug like core flow impinging upon the plate, this can be seen in figure 13. This regime experiences no evident vortex interaction prior to impingement. The large turbulence intensity experienced at this stroke length is a result of the periodic nature of the highly coherent core. In contrast to the $\mathrm{L}_{0} / \mathrm{D}=4$ in figure 6 , the large turbulence intensity extends all the way to the impingement surface.
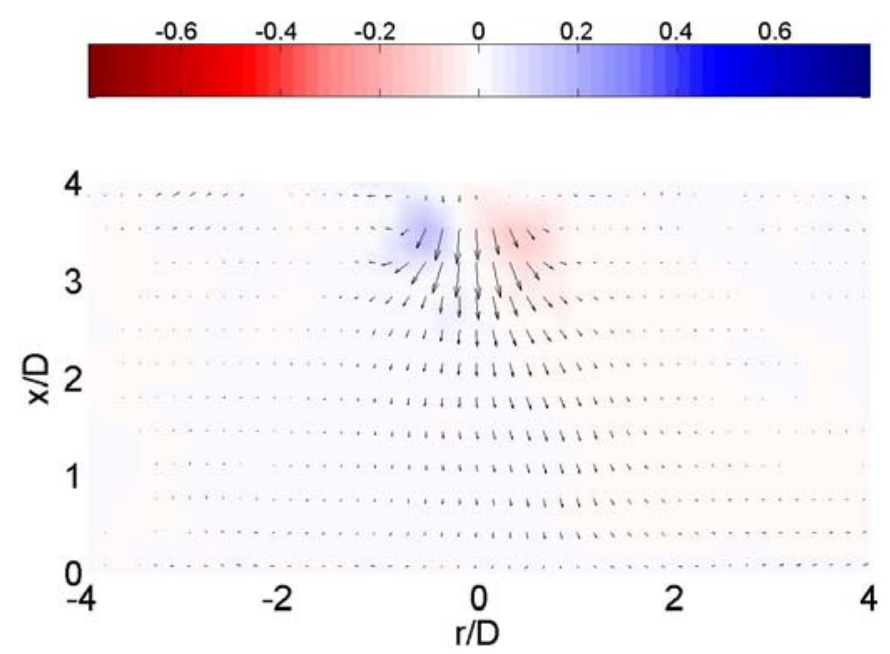

Figure 9: Phase-locked flow field and dimensionless vorticity $\omega D / U_{0}$ distribution of impinging synthetic jet at the end of the ejection phase $(\mathrm{H} / \mathrm{D}=4$, $\mathrm{L}_{0} / \mathrm{D}=2, \mathrm{Re}=3000, \varphi=270^{\circ}$ )

Figure 9 shows the PIV flow field and dimensionless vorticity for $\mathrm{H} / \mathrm{D}=4, \mathrm{~L}_{0} / \mathrm{D}=2, \mathrm{Re}=3000$. The colour bar in figure 9 represents normalised vorticity $\omega D / U_{0}$, where blue signifies clockwise movement, and red anti-clockwise. This plot helps to illustrate the fact that the synthetic jet is just about forming, i.e. the vortex has not been able to propagate away from the orifice, and the formation vortex ring shown in figure 9 after full ejection of the synthetic jet is unable to escape the near vicinity of the orifice and is reingested into the orifice during the suction phase of the synthetic jet cycle. Shuster and Smith (2007) reported that no vortices were phase locked to the actuating driver frequency at $1 \leq \mathrm{L}_{0} / \mathrm{D} \leq 3$; the results presented in figure 9 are in agreement with this finding. This effect is known to occur at non-dimensional stroke lengths of $\mathrm{L}_{0} / \mathrm{D}<2$, which is close to the limiting condition for vortex formation and advection. For values of $\mathrm{L}_{0} / \mathrm{D} \geq 2$ it was found that the synthetic jet achieved higher heat transfer than the synthetic jets formed at $\mathrm{L}_{0} / \mathrm{D}<2$ for the same jet Reynolds numbers.

The flow field presented in figure 10 corresponds with the Nusselt number profile in figure 4 for $\mathrm{L}_{0} / \mathrm{D}=16$. Greatest heat transfer is experienced at these parameters. The flow structure seen in figure 10 highlights the almost discrete nature of each period of the jet at this $\mathrm{L}_{0} / \mathrm{D}$; it is observed that no significant interactions between ejections occur.

During each ejection phase of the cycle the flow impacts as a single slug with a much greater length scale than that of the height above the surface (H/D). This type of coherent core impingement results in a significantly higher velocity upon impact and the corresponding elevation in heat transfer observed (figure 4).

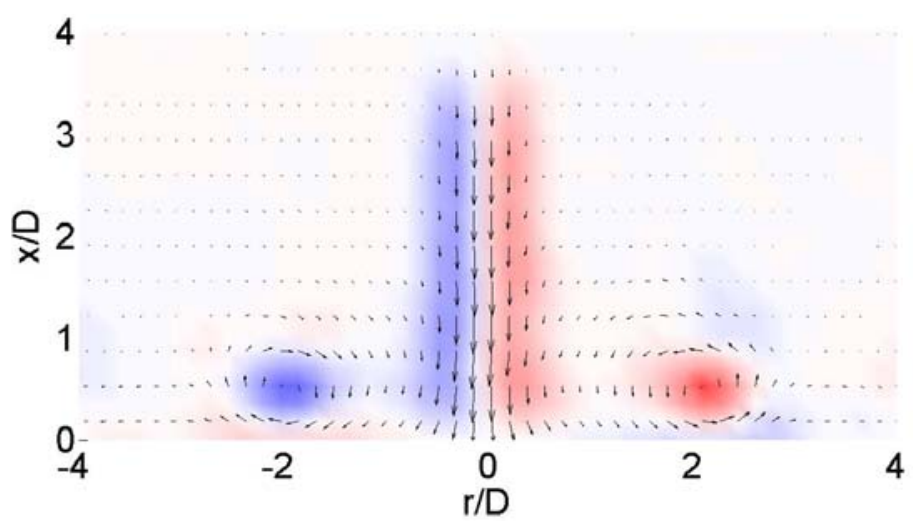

Figure 10: Phase-locked flow field and dimensionless vorticity $\omega D / U_{0}$ distribution of impinging synthetic jet at the end of the ejection phase $(\mathrm{H} / \mathrm{D}=4$, $\mathrm{L}_{0} / \mathrm{D}=16, \operatorname{Re}=3000, \varphi=270^{\circ}$ ) 


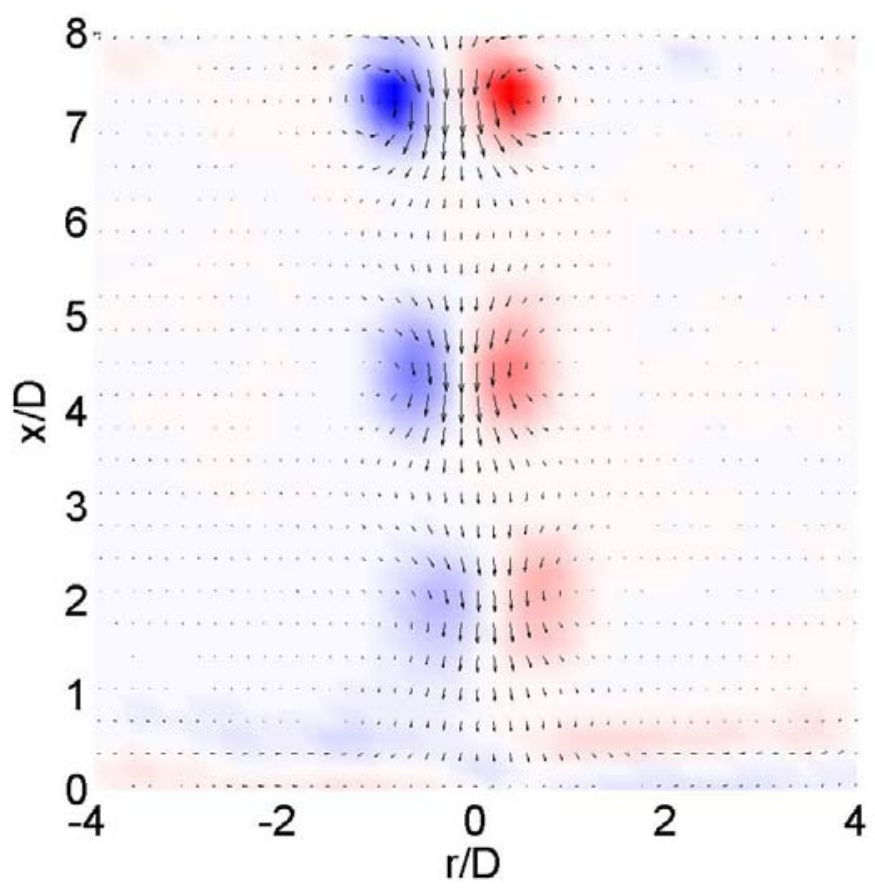

Figure 11: Phase-locked flow field and dimensionless vorticity $\omega D / U_{0}$ distribution of impinging synthetic jet at the end of the ejection phase $(H / D=8$, $\mathrm{L}_{0} / \mathrm{D}=4, \mathrm{Re}=2000, \varphi=270^{\circ}$ )

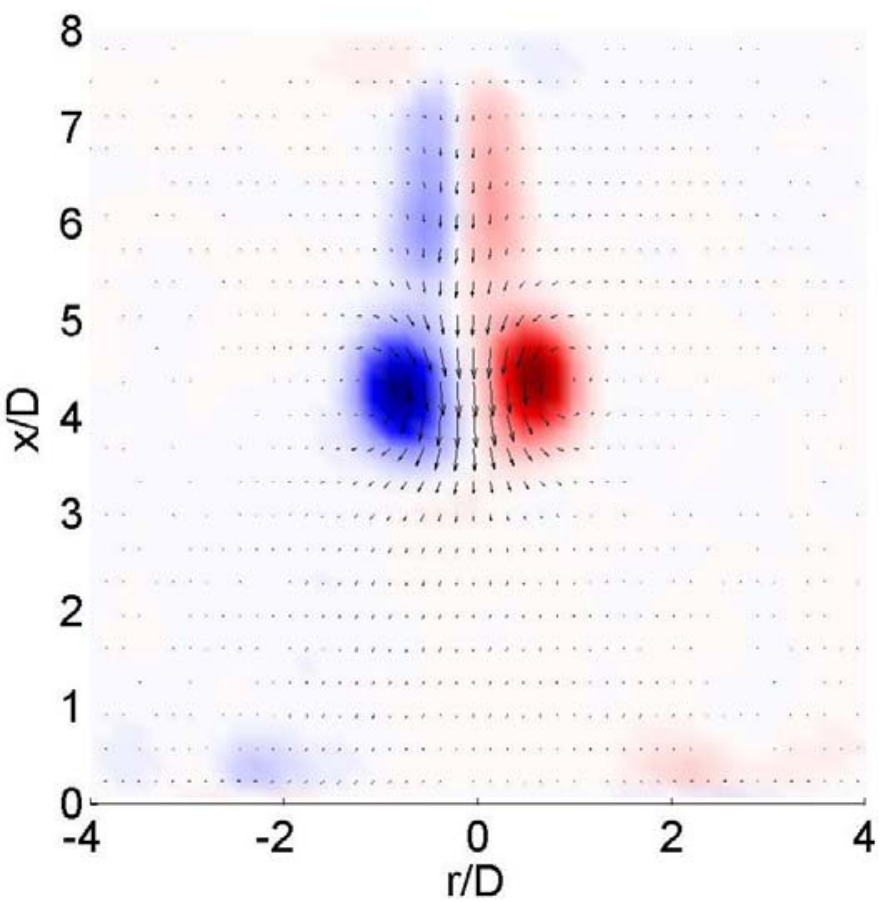

Figure 12: Phase-locked flow field and dimensionless vorticity $\omega D / U_{0}$ distribution of impinging synthetic jet at the end of the ejection phase $(\mathrm{H} / \mathrm{D}=8$, $\mathrm{L}_{0} / \mathrm{D}=8, \operatorname{Re}=2000, \varphi=270^{\circ}$ )

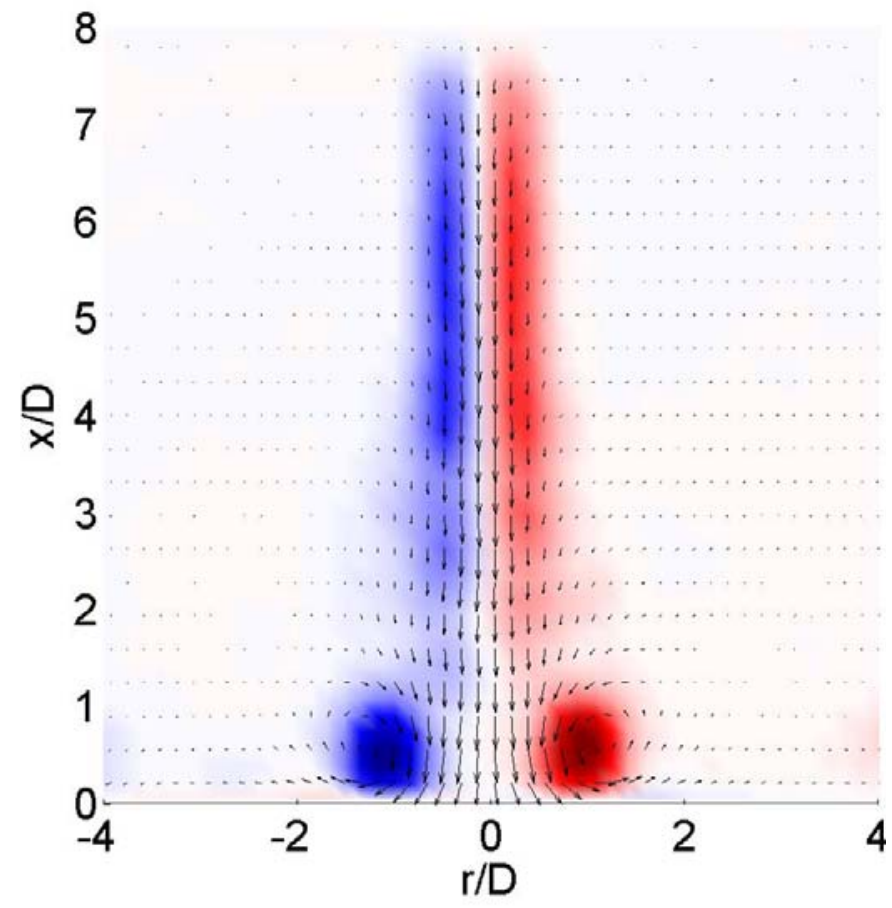

Figure 13: Phase-locked flow field and dimensionless vorticity $\omega D / U_{0}$ distribution of impinging synthetic jet at the end of the ejection phase $(H / D=8$, $\mathrm{L}_{0} / \mathrm{D}=16, \mathrm{Re}=3000, \varphi=270^{\circ}$ )

\section{CONCLUSIONS}

Stagnation point heat transfer data and Nusselt number distributions have been presented for an impinging synthetic jet. Flow field data has also been presented in the form of time averaged velocity and turbulence intensity distribution, and phase-locked flow field and dimensionless vorticity distributions for H/D values of 4 and 8 , and Reynolds numbers 1000 to 3000 and $\mathrm{L}_{0} / \mathrm{D}$ of 2 to 16 .

Four different stagnation point heat transfer regimes were identified and investigated using corresponding radial heat transfer profiles and flow field data.

Results obtained showed a strong correlation between the heat transfer data and the flow field data; specifically that of the interactions of the vortices at low $\mathrm{L}_{0} / \mathrm{D}$ values and the effective heat transfer. It was also noted that a slug flow regime was identified at large stroke lengths which also provided increased heat removal rates.

Similar trends to those identified in this paper have been observed by authors such as Valiorgue et al. (2008) and Shuster and Smith (2007). 


\section{ACKNOWLEDGMENTS}

The authors acknowledge the technical support staff of the Mechanical and Manufacturing Engineering Department of Trinity College Dublin.

The project is part funded by Science Foundation Ireland (SFI) grant number 07/RFP/ENMF220, the Centre for Telecommunications Value-Chain Research (CTVR), and Irish Research Council for Science, Engineering and Technology (IRCSET).

\section{REFERENCES}

Black W.Z., Glezer A., Hartley J.G., (1998) "Heat Transfer Modules for Cooling Electronics Packages" International Symposium on Advanced Packaging Materials

Gardon R., Akfirat J.C., (1965) "The role of turbulence in determining the heat-transfer characteristics of impinging jets" International Journal of Heat and Mass Transfer, Volume 8, Issue 10, October 1965, Pages 1261-1272

Gillespie M., Black W., Rinehart, C., Glezer, A., (2006) "Local Convective Heat Transfer from a Constant Heat Flux Flat Plate Cooled by Synthetic Air Jets" ASME Journal of Heat Transfer, Vol. 128.

Glezer A., Amitay M., (2002) "Synthetic Jets" Annual Review Fluid Mechanics, 34:503-29.

Holman R., Utturkar Y., Mittal R., Smith B. L., Cattafesta L., (2005) "Formation Criterion for Synthetic Jets" AIAA Journal Vol. 43, No. 10.

Hollworth B.R., Wilson S.I., (1984) "Entrainment effects on impingement heat transfer I: Measurements of heated jet velocity and temperature distributions and recovery temperatures on target surface" Journal of Heat Transfer, vol. 106, $n^{\circ} 4$, pp. 797-803

Kercher D. S., Lee J. B., Brand O., Allen M. G., Glezer A., (2003) "Microjet cooling devices for thermal management of electronics" J. Chem. Theory Comput., 26_2_, pp. 359-366.

Lyttle D., Webb B., 1994. “Air jet impingement heat transfer at low nozzle-plate spacings" Int. J. Heat Mass Transfer 37, pp. 1687-1697.

McGuinn A., Persoons T., O’Donovan T. S., Murray D. B., (2009) "Heat transfer and air temperature measurements of an impinging synthetic air jet" Turbulence, Heat and Mass Transfer 6, Begell House Inc. New York.
O'Donovan T. S., Murray, D. B., (2007) "Jet impingement heat transfer - Part I: Mean and root-mean-square heat transfer and velocity distributions," International Journal of Heat and Mass Transfer, vol. 50, pp. 3291-3301.

Pavlova, A. and Amitay, M., (2006) "Electronic Cooling Using Synthetic Jet Impingement" ASME Journal of Heat Transfer, 128 pp 897 - 907.

Persoons T., O’Donovan T. S., (2007) “A pressure-based estimate of synthetic jet velocity" Physics of Fluids 19(12):128104.

Smith B. L., Glezer A., (1998) "The formation and evolution of synthetic jets" Physics of Fluids 10:2281-2297.

Shuster J. M., Smith D. R., (2007) "Experimental study of the formation and scaling of a round synthetic jet" Physics of Fluids, 19:045109

Valiorgue P., Persoons T., McGuinn A., Murray D. B., (2008) "Heat transfer mechanisms in an impinging synthetic jet for a small jet-to-surface spacing," Experimental Thermal and Fluid Science, vol. 33, pp. 597-603 